\title{
Etiologic profile of breast dermatoses in Dakar: A prospective study on 125 cases
}

\section{Boubacar Ahy Diatta, Harakat Hind, Ndéye Bougoul Seck, Saer Diadie, Aminata Deh, Khadim Diop, Doudou Diouf, Mame Téning Ndiaye, Assane Diop, Maodo Ndiaye, Moussa Diallo, Fatimata Ly, Suzanne Oumou Niang}

Department of Dermatology of the Cheikh Anta Diop University of Dakar, Daakar, Senegal

Corresponding author: Dr. Boubacar Ahy Diatta, E-mail: ahydiatta@yahoo.com

\begin{abstract}
Background: The severity of breast dermatoses is related to the existence of an underlying malignant neoplasia with a risk of mastectomy that involves both vital and functional prognosis. The aim of this study was to determine the epidemiological, clinical and etiological aspects of breast dermatoses in Dakar. Materials and methods: It was a multicenter descriptive study over a 6-month period in three departments in Dakar. All patients who were referred for dermatosis localized only in the breast were included in this study. Results: We collected 125 cases, a hospital frequency of $1.02 \%$. The sex ratio was 0.016 . The average age was 45 years [16 -79 years]. The dermatoses of the breast were tumoral in 92 cases, infectious in 25 cases and immuno-allergic in 8 cases. The tumoral pathologies were malignant in 90 cases and benign in 2 cases. Metastases were pulmonary ( 32 cases), ganglionic ( 29 cases), hepatic (14 cases), bony (13 cases), renal (1 cases) and cerebral (1 cases). The treatment was etiological with topical corticosteroids, antibiotics and antifungals. The different treatments for the tumors were surgery, chemotherapy, hormone therapy and radiotherapy. A cure was noted in 26 cases (20.8\%) and one death in 6 cases. Conclusion: We report a high prevalence of breast cancer in adult women and the occurrence of mastitis and puerperal breast abscess in young women and a high frequency of allergic contact dermatitis during atopy.
\end{abstract}

Key words: Etiology; Breast dermatoses; Dakar

How to cite this article: Diatta BA, Hind H, Seck NB, Diadie S, Deh A, Diop K, Diouf D, Ndiaye MT, Diop A, Ndiaye M, Diallo M, Ly F, Niang SO. Etiologic profile of breast dermatoses in Dakar: A prospective study on 125 cases. Our Dermatol Online. 2019;10(3):244-250.

Submission: 19.03.2019; Acceptance: 18.05.2019

DOI:10.7241/ourd.20193.4 


\title{
Profil étiologique des dermatoses du sein à Dakar: Une étude prospective sur 125 cas
}

\section{Boubacar Ahy Diatta, Harakat Hind, Ndéye Bougoul Seck, Saer Diadie, Aminata Deh, Khadim Diop, Doudou Diouf, Mame Téning Ndiaye, Assane Diop, Maodo Ndiaye, Moussa Diallo, Fatimata Ly, Suzanne Oumou Niang}

\author{
Department of Dermatology of the Cheikh Anta Diop University of Dakar, Daakar, Senegal
}

Corresponding author: Dr. Boubacar Ahy Diatta, E-mail: ahydiatta@yahoo.com

\begin{abstract}
RÉSUMÉ
Introduction: La gravité des dermatoses du sein est liée à l'existence d'une néoplasie maligne sous-jacente avec un risque de mastectomie qui met en jeu le pronostic vital et fonctionnel. Lobjectif de cette étude était de déterminer les aspects épidémiologiques, cliniques et étiologiques des dermatoses du sein à Dakar. Matériels et méthodes: Il s'agissait d'une étude descriptive multicentrique sur une période de 6 mois dans trois services à Dakar. Tous les patients venus consulter pour une dermatose localisée uniquement au niveau de la région mammaire ont été inclus dans l'étude. Résultats: Nous avons colligé 125 cas soit une fréquence hospitalière de 1,02\%. Le sexe ratio était de 0,016. La moyenne d'âge était de 45 ans [16 -79 ans]. Les dermatoses du sein étaient tumorales dans 92 cas, infectieuses dans 25 cas et immuno-allergiques dans 8 cas. Les pathologies tumorales étaient malignes dans 90 cas et bénignes dans 2 cas. Les métastases étaient pulmonaires (32 cas), ganglionnaires (29 cas), hépatiques ( 14 cas), osseuses ( 13 cas), rénales ( 1 cas) et cérébrales (l cas). Le traitement était étiologique avec les dermocorticoïdes, les antibiotiques et les antifongiques. Les différents traitements des tumeurs étaient la chirurgie, la chimiothérapie, l'hormonothérapie et la radiothérapie. Une guérison était notée dans 26 cas $(20,8 \%)$ et un décès dans 6 cas. Conclusion: Notre rapportons une prévalence élevée du cancer du sein chez les femmes adultes et la survenue des mastites et des abcès du sein puerpérales chez la femme jeune et une plus grande disposition à développer un eczéma de contact en cas de dermatite atopique.
\end{abstract}

Mots clés: Etiologies; Dermatoses du sein; Dakar

\section{INTRODUCTION}

Les dermatoses du sein regroupent l'ensemble des manifestations dermatologiques localisées à la glande mammaire et/ou de son revêtement cutané ou secondaire à une maladie générale. Les causes de l'atteinte spécifique de la peau du sein sont multiples d'origine le plus souvent infectieuse, tumorale et/ou immunoallergique [1]. La gravité des dermatoses mammaires est liée d'une part à l'existence d'une néoplasie maligne sous-jacente avec une possibilité de mise en jeu du pronostic vital par le biais d'une métastase à distance et d'autre part au préjudice fonctionnel par le risque de mastectomie et d'arrêt de l'allaitement [2-5]. Peu d'études ont rapporté la prévalence et les différentes causes des dermatoses du sein en Afrique subsaharienne alors que le cancer du sein occupe la première cause des pathologies mammaires au Sénégal [6]. Lobjectif de cette étude était de déterminer les aspects épidémiologiques, cliniques et étiologiques des dermatoses du sein à Dakar.

\section{MATÉRIELS ET MÉTHODES}

Il s'agissait d'une étude descriptive multicentrique sur une période de 6 mois dans les services de Dermatologie et de cancérologie de l'Hôpital Aristide Le Dantec et le service de Dermatologie de l'Institut d'Hygiène Sociale. Nous avons inclus tous les patients venant consulter pour une dermatose localisée uniquement au niveau de la région mammaire.

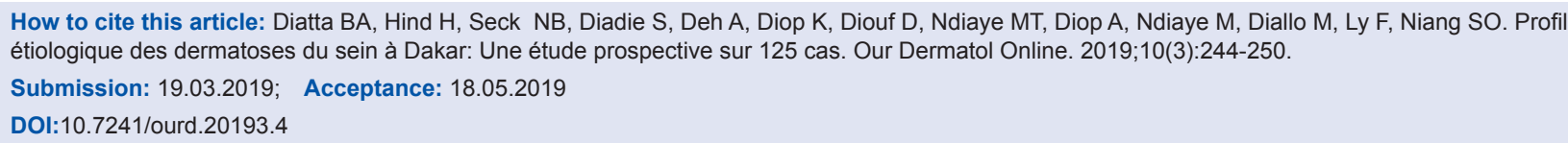


Nous avons exclus les patients qui présentaient une dermatose mammaire relevant d'une maladie générale. Le diagnostic de dermite de contact était basé sur l'interrogatoire (recherche d'une notion d'application de produits), l'examen clinique et l'exploration allergologique par les patch-tests. Le diagnostic de dermatite atopique est basé sur l'interrogatoire à la recherche d'équivalent atopique familial ou personnel (asthme, rhinite allergique, conjonctivite allergique, eczéma) et les critères diagnostiques de l'United kingdom working party. Le diagnostic de l'infection était basé sur les signes cliniques évocateurs et les explorations paracliniques notamment mycologiques, bactériologiques et à l'échographie.

Les causes tumorales étaient confirmées par l'examen anatomo-pathologique soit par le biais d'une biopsie cutanée ou au tru-cut. Léchographie et la mammographie étaient demandées à la recherche de lésions suspectes de malignité. Le scanner thoracoabdomino-pelvien recherchait une localisation secondaire. Les explorations allergologiques étaient effectuées avec la batterie standard européenne. La saisie et l'analyse des données ont été effectuées sur les logiciels Sphinx version 5.1.0.2 et SPSS version 18.

\section{RÉSULTATS}

Nous avons recensé 125 cas de dermatoses mammaires sur 12211 malades vus durant la période de l'étude soit une fréquence hospitalière de $1,02 \%$. Le sexe ratio était de 0,016 soit 2 hommes et 123 femmes. La moyenne d'âge était de 45 ans avec des extrêmes de 16 ans à 79 ans. La répartition des tranches d'âge selon les groupes pathologiques est illustrée sur la Fig. 1. Le délai moyen de consultation était de 8 mois avec des extrêmes de 5 jours à 5 ans.

Les circonstances de découverte étaient la douleur (18 cas), le prurit(8 cas), le placard inflammatoire (25 cas), l'intertrigo (10 cas), l'érythème (2 cas) et une masse mammaire (62 cas). Les malades ont eu recours en première intention au médecin généraliste dans 49 cas et à un tradipraticien dans 25 cas. Les antécédents ou terrains étaient cardio-vasculaires (une hypertension artérielle (12 cas), un diabète (6 cas), une dyslipidémie (2 cas) et une obésité (8 cas), immuno-allergiques (une atopie dans 9 cas et un eczéma des seins dans 4 cas), infectieuses (un abcès dans 2 cas, un intertrigo sous mammaire dans 2 cas) et tumorales (carcinome canalaire infiltrant dans 4 cas). L'état général était conservé dans 118 cas et altéré dans 7 cas. Une fièvre était notée dans 6 cas. Les lésions mammaires étaient unilatérales dans 107 cas et bilatérales dans 18 cas.

Les différents aspects cliniques lésionnels sont répertoriés sur le Tableau I. Nous avons noté trois groupes

\begin{tabular}{lcc} 
Tableau I : Répartition des cas en fonction des lésions mammaires \\
\hline Aspects des lésions & Effectifs & $\%$ \\
\hline Erythème & 82 & 65,6 \\
Masse tumorale & 63 & 50,4 \\
Induration & 42 & 33,6 \\
Aspect peau d'orange & 36 & 28,8 \\
Rétraction & 31 & 24,8 \\
Ecoulement mamelonnaire & 16 & 12,8 \\
Nécrose & 10 & 8 \\
Enduit blanchâtre & 9 & 7,2 \\
Nodule & 8 & 6,4 \\
Vésicules & 6 & 4,8 \\
Troubles pigmentaires & 5 & 4 \\
Lichénification & 2 & 1,6 \\
\hline
\end{tabular}

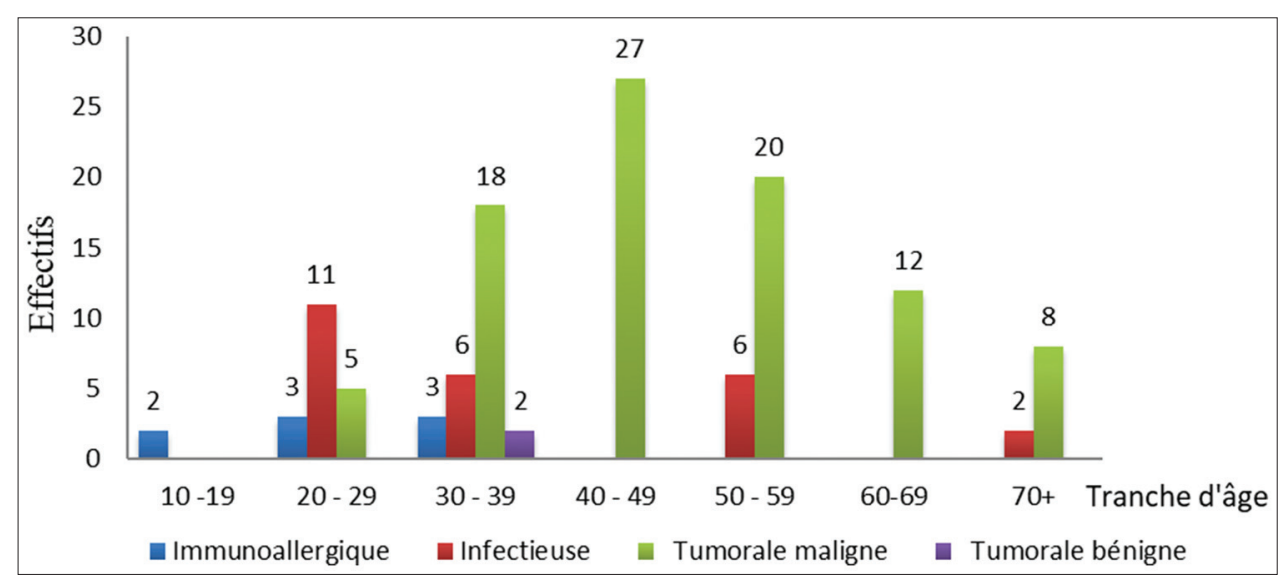

Figure 1: Répartition des tranches d'âge en fonction des groupes pathologiques. 
de pathologies mammaires. Il s'agissait de pathologies tumorales dans $73,6 \%$ (92 cas), infectieuses dans $20 \%$ ( 25 cas) et immuno-allergiques dans 6,4\% (8 cas). Les pathologies tumorales étaient malignes dans 90 cas et bénignes dans 2 cas.

La répartition des cas en fonction des types de tumeurs est illustrée sur le Tableau II.

Les pathologies infectieuses étaient bactériennes dans $60 \%$ ( 15 cas) à type d'abcès du sein (9 cas) (Fig. 2), de mastites puerpérales (6 cas) et d'origine mycosique (10 cas).

Les pathologies immuno-allergiques étaient à type de dermite de contact allergique (7 cas) (Fig. 3) et de dermatite atopique (l cas).

La topographie des lésions au niveau mammaire était au niveau des 4 cadrans dans 52 cas, la zone périaréolaire dans 19 cas, la région sous mammaire dans 10 cas, les aréoles dans 2 cas, les mamelons dans 3 cas, la région pariétale dans 4 cas.

Les adénopathies étaient présentes dans 89 cas soit $70,6 \%$ avec un aspect.

Inflammatoire dans 10 cas et non inflammatoire dans 79 cas.

Ils siégeaient aux niveaux axillaires dans 89 cas et sus claviculaires dans 10 cas. Les métastases notées dans 47 cas étaient pulmonaires (32 cas), ganglionnaires (29 cas), hépatiques (14 cas), osseuses (13 cas), rénales (1 cas) et cérébrales dans 1 cas.

L'histologie a confirmé un carcinome épidermoïde dans 2 cas, un molluscum pendulum (Fig. 4), Une mastite et un lymphome non hodgkinien dans 1 cas.

Tableau II : La répartition des cas en fonction des types de tumeurs malignes

\begin{tabular}{lccc}
\hline Types de tumeurs & Effectifs & & $\%$ \\
\hline Bénignes & 1 & 0,8 & 1,6 \\
$\quad$ Adénofibrome & 1 & 0,8 & \\
$\quad$ Molluscum pendulum & & & 72 \\
Malignes & & & \\
Carcinome canalaire & 49 & 39,2 & \\
$\quad$ Carcinome infiltrant & 23 & 18,4 & \\
CCl & 6 & 4,8 & \\
Adénocarcinome & 4 & 3,2 & \\
$\quad$ Récidive tumorale & 3 & 2,4 & \\
Carcinome lobulaire & 3 & 2,4 & \\
Carcinome épidermoïde & 1 & 0,8 & \\
Carcinome mucineux & 1 & 0,8 & \\
Lymphome & &
\end{tabular}

(c) Our Dermatol Online 3.2019
La biopsie mammaire au tru-cut a été effectuée dans 86 cas,elle a permis de confirmer un carcinome infiltrant (49 cas) (Fig. 5), un carcinome canalaire infiltrant dans 23 cas,un adénocarcinome dans 6 cas, un carcinome lobulaire dans 3 cas, une mastite aigue dans 2 cas,

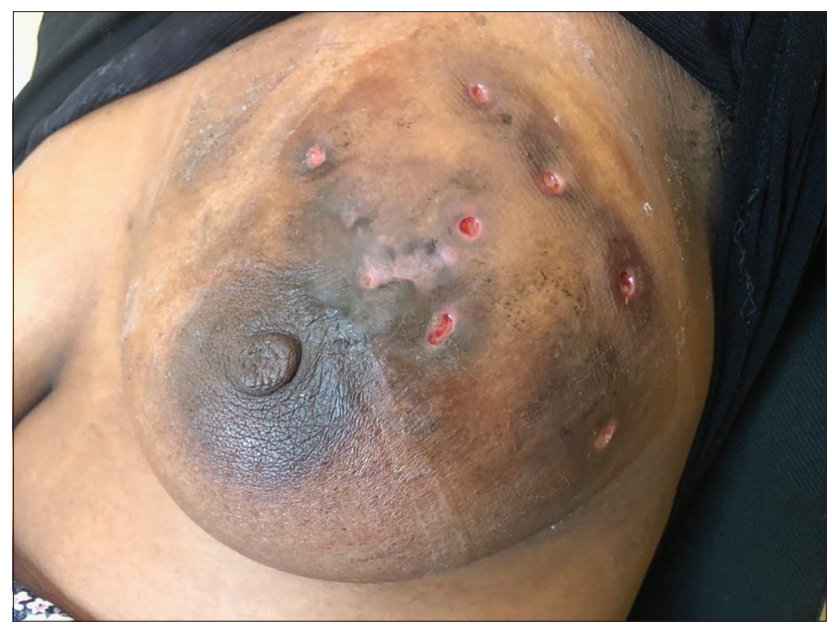

Figure 2: Un abcès du sein fistulisé chez une nourrice de 25ans.

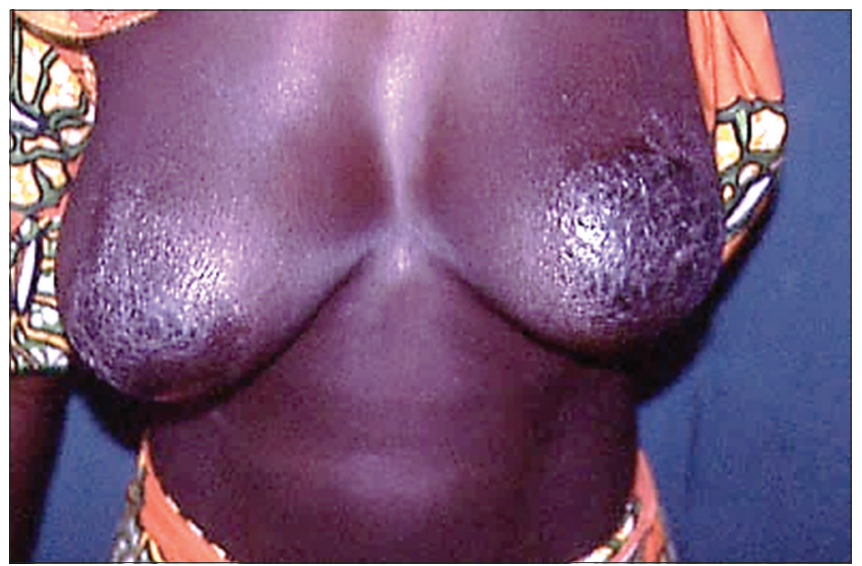

Figure 3: Eczéma de contact des seins au bichromate de potassium et fragrance mix.

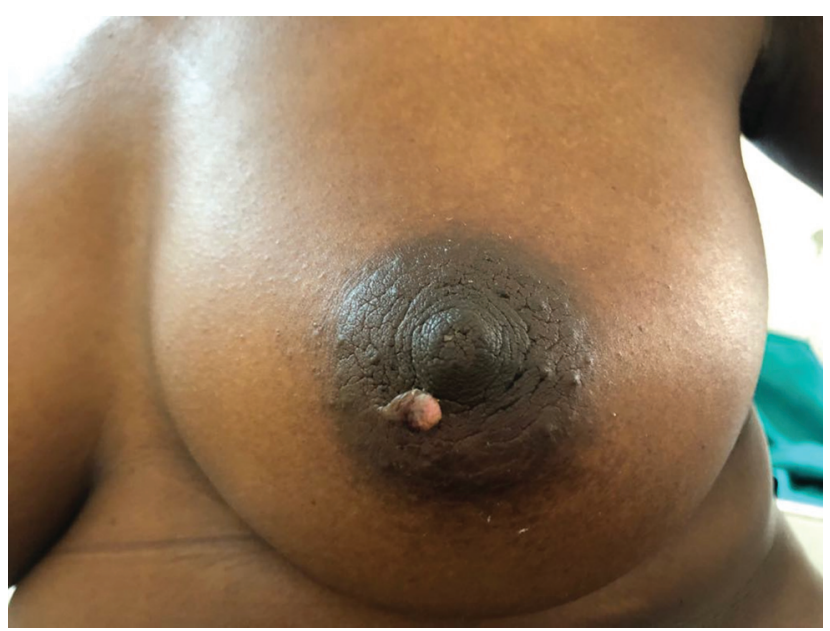

Figure 4: Molluscum pendulum chez une femme de 35 ans. 


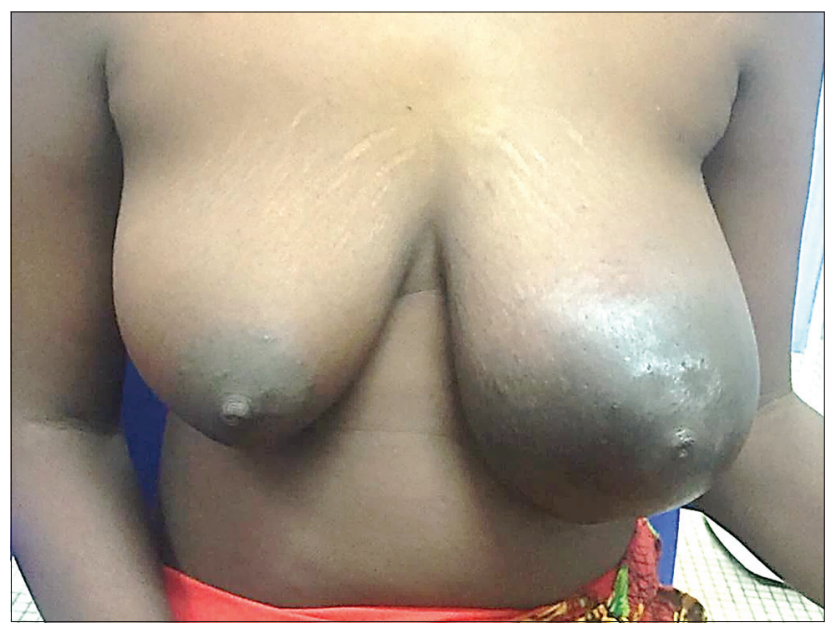

Figure 5: Un carcinome infiltrant inflammatoire du sein gauche.

un carcinome épidermoïde dans 1 cas, un carcinome mucineux dans 1 cas et une tumeur conjonctive bénigne dans 1 cas. Lexamen mycologique a isolé le Candida albicans dans 2 cas. Les patch-tests étaient positifs au bichromate de potassium et le paraphénylénediamine dans 2 cas. Le bichromate de potassium et le fragrance mix 1 dans 1 cas. Le fragrance mix 1 et la benzocaïne dans 1 cas. Le bichromate de potassium et le clioquinol dans $l$ cas.

L'échographie mammaire a décelé une formation tissulaire suspecte de malignité dans 48 cas. La mammographie était réalisée dans 51 cas.Selon la classification de l'American College of Radiology (ACR), les malades étaient classés en ACR 1 (2 cas), ACR 2 ( 4 cas), ACR 3 (3 cas), ACR 4 (10 cas) et ACR 5 (32 cas). Le scanner thoraco-abdomino-pelvien réalisé dans $80 \mathrm{cas}$, a permis de détecter des localisations métastasiques secondaires dans 47 cas $(52,2 \%)$.

Le traitement était celui de la cause. Les dermocorticoïdes et les antihistaminiques dans l'eczéma de contact (8 cas). Les antifongiques dans les infections mycosiques ( 15 cas), les antibiotiques dans 15 cas d'infections bactériennes associés à un drainage d'abcès dans 9 cas.

Pour les causes tumorales une chimiothérapie a été utilisée en première ligne avec le protocole $\mathrm{AC}$ (Anthracycline + cyclophosphamide) dans 59 cas. Le protocole FAC (5-Fluoro-Uracile + anthracycline + cyclophosphamide) dans 13cas. Les taxanes étaient utilisés en $2^{\text {ème }}$ ligne dans 15 cas.L'hormonothérapie (anastrazole) était utilisée dans 2 cas. Le traitement chirurgical consistait à une tumorectomie dans 2 cas, une mastectomie dans 1 cas et une biopsie exérèse dans 1 cas. La radiothérapie était utilisée dans 2 cas. Lévolution était favorable pour les causes infectieuses dans 17 cas, pour les causes immuno-allergiques dans 7 cas et les causes tumorales dans 2 cas. Une réponse tumorale partielle était notée dans 9 cas et un décès dans 6 cas secondaire au cancer du sein.

En analyse bi varié nous avons noté une corrélation significative entre l'âge adulte et la survenue du cancer du sein et l'existence d'une atopie au cours de l'eczéma de contact.

\section{DISCUSSION}

Notre étude rapporte une fréquence hospitalière de $1,02 \%$ des dermatoses mammaires à Dakar. Ces dermatoses se caractérisent par une prédominance des causes tumorales dans $72 \%$, suivi des causes infectieuses et immunoallergiques.

Au Sénégal le cancer du sein et de l'appareil gynécologique occupent le premier rang et représentent $54 \%$ des cancers de la femme selon le registre des tumeurs [6].

Par ordre de fréquence, les tumeurs malignes étaient réparties en carcinomes canalaires $(91,1 \%)$, en carcinome lobulaire $(3,3 \%)$, en carcinome épidermoïde $(3,3 \%)$, en carcinome mucineux $(1,1 \%)$ et en lymphome non hodgkinien $(1,1 \%)$.

La prédominance des carcinomes canalaires parmi les types histologiques de cancer du sein est concordante avec les résultats rapportés dans la littérature $[7,8]$. Les carcinomes épidermoïdes primitifs du sein ou carcinomes à cellules squameuses sont rares et représentent 0,1 à $2 \%$ de l'ensemble des carcinomes du sein, ils appartiennent au groupe hétérogène des carcinomes métaplasiques mammaires et sont d'étiopathogénie et de pronostic controversés [9]. Le carcinome du sein peut interpeller le dermatologue à différents stades de son évolution. Les principales modifications cutanées induites par le cancer du sein sont: le placard inflammatoire, la rétraction du mamelon, l'induration, l'aspect en peau d'orange ou encore l'ulcération [10-12].

Les dermatoses infectieuses occupaient le second rang des affections mammaires. Les mastites étaient trouvées dans 6 cas soit $4,8 \%$ et les abcès du sein dans 9 cas soit $7,2 \%$, ils étaient tous d'origine puerpérale. 
Deux formes principales de mastites sont décrites dans la littérature: les mastites puerpérales survenant pendant les périodes de lactation, et les mastites non puerpérales.

La mastite puerpérale touche $10-20 \%$ des femmes pendant les six premiers mois de lactation et le risque de développer un abcès en cas de mastite est de 5-10\%. La stase laiteuse constitue un facteur de risque pour le développement d'une infection bactérienne des canaux lactifères et lymphatiques, particulièrement en présence d'excoriations au niveau du mamelon [16]. Quant à l'abcès du sein, sa fréquence était plus élevée que celle rapportée dans la littérature: 3\% aux Etats Unis et Allemagne [13-15] et de 0,52\% en France [9]. Les causes immunoallergiques occupaient le troisième rang, il s'agissait de 7 cas d'eczéma de contact et d'un cas de dermatite atopique.

Leczéma de contact était associé à un terrain atopique dans 5 cas soit $71,4 \%$ avec un lien statistiquement significatif. Ceci rejoint les données de la littérature qui montre une prévalence plus élevée de l'eczéma de contact du sein avec un terrain d'atopie [17]. Les eczémas de contact au bichromate de potassium peuvent être liés à la composition du textile. Certaines industries du textile utilisent cette substance pour la teinture du coton et la laine notamment pour le linge du corps ou les vêtements et peuvent ainsi être la source allergénique. Le PPD est un colorant noir qui entre dans la composition de certains colorants textiles utilisés dans la fabrication des vêtements ou du linge du corps.

De ce fait, il peut être la source allergénique à l'origine de l'eczéma au PPD.

Le fragrance mix 1 est un mélange de 8 allergènes servant à dépister une allergie aux parfums. On peut le retrouver dans divers produits cosmétiques: parfums, eau de toilette, maquillage, lait de corps [18-20].

Une évolution favorable était notée dans 68\% parmi les causes infectieuses, $87,5 \%$ des causes immunoallergiques et 2,1\% des causes tumorales. Il s'agissait de tumeurs bénignes (molluscum pendulum et adénofibrome) qui avaient reçu une tumorectomie. Une réponse partielle avec diminution de la taille de la tumeur sous chimiothérapie était notée dans 9,8\% des cas des causes tumorales.

Une persistance de la tumeur était notée dans 19,6\% des cas des causes tumorales.
Cependant le recul était insuffisant pour apprécier l'efficacité définitive de la chimiothérapie.

\section{CONCLUSION}

Les manifestations dermatologiques mammaires peuvent être primitives ou secondaires à une maladie sous jacente. Notre étude rapporte une prévalence élevée du cancer du sein chez les femmes adultes entre 40 et 50 ans. La fréquence des mastites et des abcès du sein au cours de la période puerpérale chez les jeunes femmes âgées entre 20 et 30 ans et une plus grande disposition à développer un eczéma de contact en cas de dermatite atopique.

\section{Statement of Human and Animal Rights}

All procedures followed were in accordance with the ethical standards of the responsible committee on human experimentation (institutional and national) and with the Helsinki Declaration of 1975, as revised in 2008 .

\section{Statement of Informed Consent}

Informed consent was obtained from all patients for being included in the study.

\section{RÉFÉRENCES}

1. Levy-Bencheton A, Agostini A. Dermatoses de l'aréole et du mamelon. Encycl Méd Chir. 2013;98-871-A-10.

2. Lachapelle JM, Borradori L. Dermatologie et infections sexuellement transmissibles Gème édition: Dermatoses des seins: 958-61.

3. Boulanger L, Demetz J. Comment explorer une lésion cutanée mammaire ?: Recommandations. J Gynecol Obst Bio R 2015;44:921-6.

4. Geffroy D, Dutriaux-Dumoulins I, Labbe-Devilliers C, Meingan P, Houdebine S, et al. Maladie de Paget du mamelon et principaux diagnostics différentiels. J Radiol. 2011;92:889-98.

5. Ryerson AB, Miller J, Eheman CR. Reported breast symptoms in the National Breast and Cervical Cancer Early Detection Program. Cancer Cause Control 2015;26:733-40.

6. Bouri NV, Ba O, Dieme JL, Mbengue M, Boye A, Fall T, et al. État des lieux du registre des tumeurs au Sénégal: bilan à 6 ans d'enregistrement en ligne. Rev Mal Respir. 2017;34:A75.

7. Darré T, Amégbor K, Sonhaye L, Kouyate M, Aboubaraki A, N'timo B, et al. Profil histo-épidémiologique des cancers du sein A propos de 450 cas observés au CHU de Lomé. Med Afr Noire. 2013;60:1.

8. Atangana Paul Jean A, Tchenté Nguefack C, Kabeyene Okono Angèle C, Totoum Fotsing C, Dina Bell E, Tayou Rachel. et al. Immunohistochemical pattern of breast cancer in Douala and Yaounde. Health Sci Dis. 2017;18:14-20.

9. Alaoui FZ, Benkirane S, Chaara H, Bouguern H, Melhouf MA. [Squamous cell carcinoma of the breast: report of 3 cases and literature review]. Pan Afr Med J. 2012;12:38. 


\section{www.odermatol.com}

10. Whitaker-Worth DL, Carlone V, Susser WS, Phelan N, GrantKels JM. Dermatologic diseases of the breast and nipple. J Am Acad Dermatol. 2000;43:733-51.

11. Chaari I, Garbaa S, Boudaya S, Chaabanne H, Masmoudi A, Boudaoura T, et al. Cancer du sein chez l'homme: importance des manifestations cutanées. Ann Dermatol Venereol. 2015;142:529.

12. Cutuli B, Le Nir CC, Serin D, Kirova Y, Gaci Z, Lemanski C, et al. Male breast cancer. Evolution of treatment and prognostic factors. Analysis of 489 cases. Crit Rev Oncol Hematol. 2010;73:246-54.

13. Foxman B, D'Arcy H, Gillespie B, Bobo JK, Schwartz K. Lactation mastitis: Occurrence and medical management among 946 breastfeeding women in the United States. Am J Epidemiol. 2002;155:103-14.

14. Heywang-Köbrunner S, Schreer I, Dershaw D, Grumbach L. Atteintes inflammatoires du sein. Imagerie de la Femme. 2005;15:40-52.

15. Dixon JM, Khan LR. Treatment of breast infection. BMJ. 2011;342:d396.

16. Amir LH, Forster D, McLachlan H, Lumley J. Incidence of breast abscess in lactating women: report from an Australian cohort. BJOG. 2004;111:1378-81.

17. Kim SK, Won Y, Kim SJ. Nipple eczema: a diagnostic challenge of allergic contact dermatitis. Ann Dermatol. 2014;26:413-4.

18. Thyssen JP, White JM. Epidemiological data on consumer allergy to p-phenylenediamine. Contact Dermatitis. 2008;59:327-43.

19. Bonnetblanc JM. Allergies cutanéo-muqueuses chez l'enfant et l'adulte: eczéma de contact. Ann Dermatol Venereol. 2012;139:A78-A84.

20. Crépy MN. Les allergènes de la batterie standard dans l'environnement professionnel et non professionnel. Doc Med Trav. 2008;113:99-117.

Copyright by Boubacar Ahy Diatta, et al. This is an open-access article distributed under the terms of the Creative Commons Attribution License, which permits unrestricted use, distribution, and reproduction in any medium, provided the original author and source are credited.

Source of Support: Nil, Conflict of Interest: None declared. 